\title{
Low levels of transforming growth factor-beta (TGF-beta) and reduced suppression of Th2-mediated inflammation in hyperreactive human onchocerciasis
}

\author{
S. KORTEN ${ }^{1,3}$, A. HOERAUF ${ }^{1}$, J. T. KAIFI ${ }^{2}$ and D. W. BÜTTNER ${ }^{3}$ \\ ${ }^{1}$ Institute for Medical Microbiology, Immunology and Parasitology, University of Bonn, Sigmund-Freud-Strasse 25, \\ D-53105 Bonn, Germany \\ ${ }^{2}$ Department of General, Visceral and Thoracic Surgery, University Medical Centre Hamburg-Eppendorf, Martinistrasse \\ 52, D-20246 Hamburg, Germany \\ ${ }^{3}$ Bernhard Nocht Institute for Tropical Medicine, Bernhard-Nocht-Strasse 74, D-20359 Hamburg, Germany
}

(Received 12 November 2009; revised 28 February and 16 May 2010; accepted 17 May 2010; first published online 12 Fuly 2010)

\section{SUMMARY}

Th2-biased inflammation with eosinophilia and $\operatorname{IgE}$ production is a hallmark of helminth infections. It is pronounced in hyperreactive onchocerciasis patients ('sowda' or 'local form'), who efficiently kill microfilariae resulting in severe dermatitis and lymphadenitis. In contrast, hyporeactive patients ('generalised form') tolerate high microfilarial loads. This is thought to be mediated by regulatory $\mathrm{CD}^{+}{ }^{+} \mathrm{T}$ cells and macrophages producing suppressive cytokines such as IL-10 and transforming growth factor-beta (TGF- $\beta$ ). We investigated whether hyperreactivity was reflected by lower local TGF- $\beta$ production, analysing stable latent TGF- $\beta 1$ expression in onchocercomas, lymph nodes and skin from hyperreactive and hyporeactive patients by immunohistochemistry. TGF- $\beta$ expression was compared with that of $\operatorname{IgE}, \operatorname{IgG} 1, \operatorname{IgG} 4$, and the antigen-presenting, $\mathrm{CD}^{+}{ }^{+} \mathrm{T}$ cell-inducing MHC class II molecule HLA-DR. TGF- $\beta$ was weakly and less frequently expressed by various cell types in onchocercomas, skin and lymph nodes from hyperreactive compared to hyporeactive patients. This applied to reactions around living and dead adult worms as well as dead microfilariae. Antigen-presenting cells strongly expressed HLA-DR in both forms, but their numbers were reduced in hyperreactive nodules. Plasma cells produced more $\operatorname{IgE}$ and $\operatorname{IgG1}$, but less of the anti-inflammatory antibody $\operatorname{IgG} 4$ in hyperreactive onchocercomas. In conclusion, hyperreactivity is linked with reduced local expression of TGF- $\beta$, HLA-DR and IgG4, which might contribute to the insufficient down-regulation of inflammation via TGF- $\beta$ - and HLA-DR-induced regulatory lymphocytes.

Key words: filariasis, Onchocerca volvulus, TGF-beta, immunosuppression, inflammation, macrophages, T-lymphocytes, plasma cells, sowda.

\section{INTRODUCTION}

In sub-Saharan Africa, Yemen and Latin America an estimated 37 million people suffer from a chronic infection with the filarial nematode Onchocerca volvulus that affects skin, the lymphatic system and eyes (river blindness). Non-productive adult worms barely elicit immune responses and disease. The pathology is caused by millions of microfilariae (mf) that are released from adult worms in subcutaneous onchocercomas and which migrate to the skin and in smaller numbers to other organs. In hyperendemic foci tens or hundreds of thousands of $\mathrm{mf}$ die every day in many patients. Murdoch et al. (1993) developed a classification scheme for onchocerciasis skin lesions and a WHO Expert Committee (1995) recommended its use. Skin and lymphatic lesions were differentiated into 'generalised' and 'local' forms of

* Corresponding author: Institute for Medical Microbiology, Immunology and Parasitology, University of Bonn, Sigmund-Freud-Strasse 25, D-53105 Bonn, Germany. Tel: +49228287 5673. Fax: +492282879573. E-mail: korten@microbiology-bonn.de onchocerciasis (WHO, 1995) and these terms were also used for the polar forms of the immunological spectrum of onchocerciasis. To avoid misunderstanding, since hyporeactive onchodermatitis is also often 'local' and hyperreactive onchodermatitis may be extended, here we will only use the terms 'hyporeactive' (synonym for 'generalised form') and 'hyperreactive' (synonym for 'local or localised form' or 'sowda') for the polar forms of the immunological spectrum (WHO, 1995).

The objective of this study was to investigate whether the immunosuppressive cytokine TGF- $\beta$ plays a role in the weak immunosuppression observed in hyperreactive patients, based on our observation that $O$. volvulus elicits strong local host cell TGF- $\beta$ production in highly infected and immunosuppressed hyporeactive patients (Korten et al. 2010). The cellular and humoral immunological defence reactions to $\mathrm{mf}$, induced by filarial antigens, are usually strong in primarily infected individuals, killing the $\mathrm{mf}$ and often causing dermatitis (WHO, 1987, 1995; Cooper et al. 2001; Brattig, 2004). In hypoendemic areas the worms die after 5-12 years.

Parasitology (2011), 138, 35-45. C Cambridge University Press 2010. The online version of this article is published within an Open Access environment subject to the conditions of the Creative Commons Attribution-NonCommercial-ShareAlike licence <http://creative commons.org/licenses/by-nc-sa/2.5/>. The written permission of Cambridge University Press must be obtained for commercial re-use. 
In meso- and hyperendemic areas new infections are acquired and the reactivity decreases with increasing worm burdens in most patients. They develop the hyporeactive, immunosuppressed form of the infection, tolerating high $\mathrm{mf}$ loads and reacting only against damaged or treated $\mathrm{mf}$ and adult worms, but they do not kill viable mf (Ottesen, 1995; Brattig, 2004). However, they kill most of the newly acquired infective larvae (WHO, 1995; Schulz-Key and Soboslay, 2000). In contrast, a minority of patients develop a chronic hyperreactive form of infection with a sustained strong immunological defence reaction that kills $\mathrm{mf}$. This leads to low $\mathrm{mf}$ loads of less than $10 \mathrm{mf} / \mathrm{mg}$ skin (mostly less than $1 \mathrm{mf} / \mathrm{mg}$ ) and severe dermal and lymphatic disease (see below in the Materials and Methods section; Büttner et al. 1982; Connor et al. 1983; WHO, 1987). Usually, also the chronic hyperreactivity ends when the adult filariae die. Or, as we observed between 1978 and 1989 in Liberia, the hyperreactive form may change to the hyporeactive form, when the worm load increases after a few years (Gallin, 1987; Büttner, unpublished data). Between the two polar forms there are many patients with intermediate forms, including most primary infections, who are able to kill $\mathrm{mf}$, but present a low degree of hyporesponsiveness. The immunological spectrum of pathology hints at mechanisms based on genetic pre-disposition and immune modulatory factors that lead to variable host reactivity to the worm antigens (King and Nutman, 1991; Hoerauf et al. 2002, 2005; Brattig, 2004; Timmann et al. 2008). Hyperreactivity is characterized by a strong local and systemic Th2-type biased immune reaction, that correlates with skin and lymph node pathology and comprises elevated serum and tissue $\mathrm{IgE}$, eosinophilia, mastocytosis, increased activated $\mathrm{CD}^{+}{ }^{+} \mathrm{T}$ helper cells, dense infiltrates with $\mathrm{T}$ cells, B cells, plasma cells, and extensive fibrous tissue generation in onchocercomas (Brattig et al. 1987; Korten et al. 1998; Timmann et al. 2003; Brattig, 2004). In hyporeactive onchocerciasis, these Th2-driven inflammatory responses are weaker and mixed with a suppressive $\operatorname{Tr} 1 / \mathrm{Th} 3$ response. Antigen-specific and non-specific immunosuppression is mediated by $\mathrm{CD}^{+}{ }^{+}$regulatory $\mathrm{T}$ ('Treg) cells and macrophages, which produce suppressive cytokines such as IL-10 and TGF- $\beta$ (Kilian and Nielsen, 1989; Doetze et al. 2000; Hoerauf et al. 2005; Li et al. 2006; Taylor et al. 2006; Korten et al. 2008).

To study whether local TGF- $\beta$ expression reflects the spectrum of the polar immune states, we analysed TGF- $\beta 1$ (denoted as TGF- $\beta$ hereafter) expression in host cells of onchocercomas, lymph nodes and skin from hyperreactive compared to hyporeactive patients. We further analysed TGF- $\beta$ expression in relation to the expression of the immunoglobulins $\mathrm{IgE}, \mathrm{IgG} 1, \mathrm{IgG} 4$ and the antigen-presenting molecule HLA-DR, which induces and activates cognate Th2 and Th3 CD4 ${ }^{+}$T cells (Muixí et al. 2008).
MATERIALS AND METHODS

\section{Diagnosis of hyperreactive patients}

The diagnosis of hyperreactive onchocerciasis was based on the criteria used previously (Büttner et al. 1982; Albiez et al. 1985; Gallin, 1987; Darge and Büttner, 1995). The following criteria had to be fulfilled by all patients of this study. (1) The disease had to exist at least for a year. Usually it had existed for several years. (2) The patients had to be $\mathrm{mf}$ carriers proven by detection of $\mathrm{mf}$ in skin snips or by a positive Mazzotti test. The mf density had to be less than $10 \mathrm{mf} / \mathrm{mg}$ of skin. In Liberia, on all patients a topical Mazzotti test with diethylcarbamazine lotion had been applied on 2 areas of $20 \times 20 \mathrm{~cm}$, one on sick and one on rather healthy skin (Kilian, 1988; Fig. 1 in Darge and Büttner, 1995), because the skin snips were often negative. A Mazzotti test was considered as positive, when a reaction with at least 20 fresh papules and usually more than 100 fresh papules was observed after 1 or 2 days. All patients in our study had shown a positive test. (3) All patients had presented signs of an acute or chronic papular onchodermatitis (APOD or CPOD as described by Murdoch et al. 1993; Murdoch, 2010) and complained of strong pruritus, confirmed by scratch marks. (4) An onchocercoma with living or dead female filariae had been excised for histology. Further criteria were the presence of a dark-coloured skin area, typical asymmetry of skin lesions, lichenified onchodermatitis (LOD by Murdoch et al. 1993), and enlarged regional lymph nodes to a size of $2-6 \mathrm{~cm}$ or more (stage 2 of Murdoch et al. 1993). The patients had usually shown several of these further signs. Untreated hyperreactive patients had shown eosinophilia of the blood, usually of more than $10 \%$. The average values of the study patients were $24 \pm$ $14 \%$ and $1600 \pm 1566$ eosinophils/ $\mu$ l blood, respectively. Except for Yemen all examined hyporeactive patients had lived in highly endemic onchocerciasis foci. They were adult individuals with long-standing infections carrying high loads of nodules and more than $40 \mathrm{mf} / \mathrm{mg}$ skin.

\section{Onchocercomas, lymph nodes and skin from patients}

Sixty-four onchocercomas from 50 untreated Liberian and Yemenite patients with hyperreactive onchocerciasis were selected from previous studies (references in Table 1). Fourty-nine of these nodules were stained for TGF- $\beta$ and compared with 82 onchocercomas from 82 untreated patients with the hyporeactive form of the disease. The total number of hyporeactive nodules, stained for TGF- $\beta$ and other markers, was 130 from 94 patients. Six hyporeactive and 3 hyperreactive patients had received 1-3 standard doses of ivermectin (IVM) during mass or individual treatment and were nodulectomized between 1 day and 2 months later. One onchocercoma 

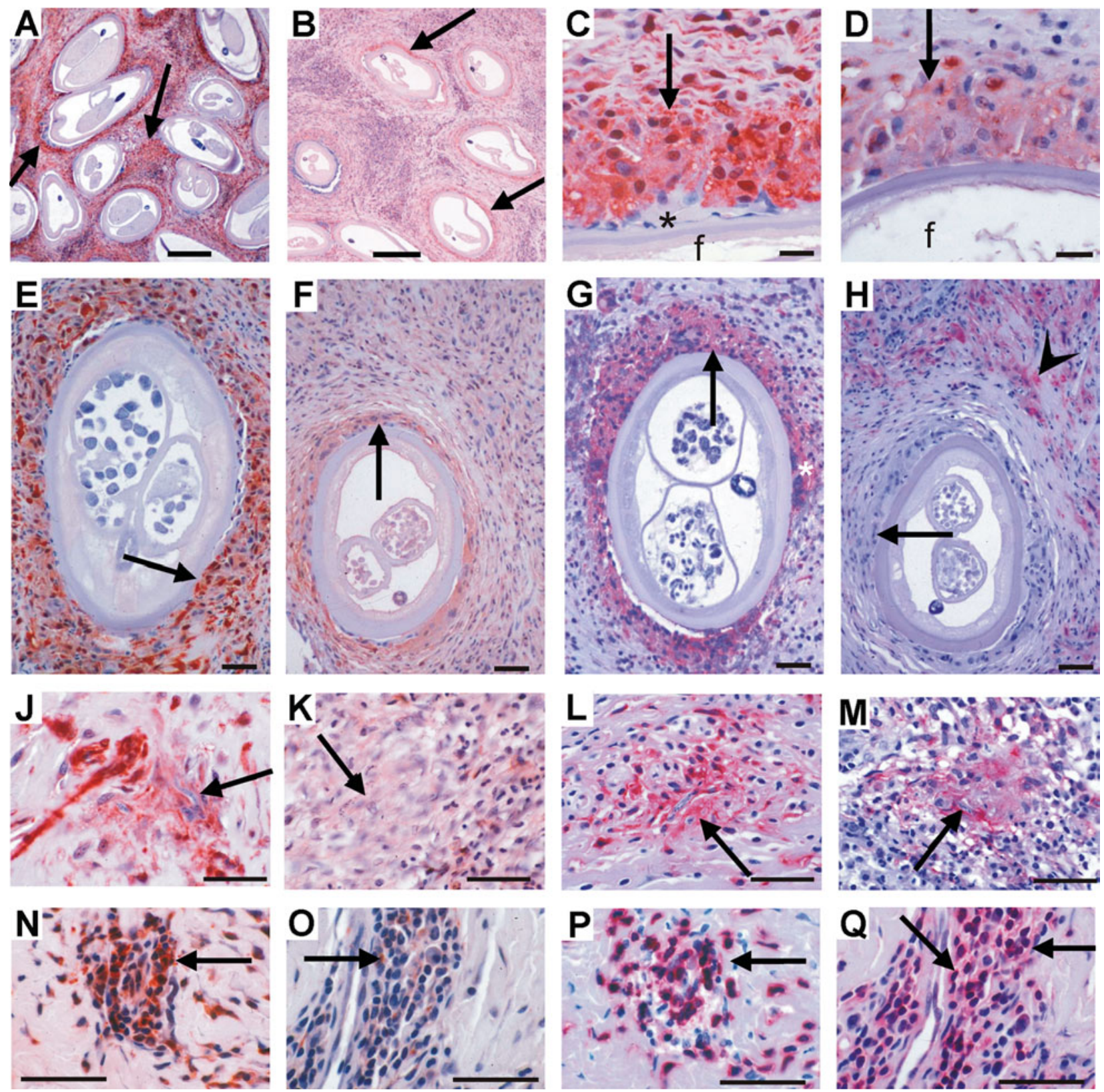

Fig. 1. Immunohistological comparison of TGF- $\beta$ and HLA-DR expression by macrophages and $\mathrm{CD}^{+}{ }^{+} \mathrm{T}$ cells in onchocercomas from untreated hyporeactive and hyperreactive patients in Liberia and Ghana. (A-B) Nodule centre with strong (hyporeactive) and weak (hyperreactive) TGF- $\beta$ expression (arrows) around older female worms. (C-D) Details of TGF- $\beta^{\text {high }}$ (hyporeactive) and TGF- $\beta^{\text {low }}$ (hyperreactive) nuclei and cytoplasm of macrophages (arrow) adjacent to female worms (f), while a small giant cell, attached to the cuticle, is negative (*). (E-H) Serial sections with strong (hyporeactive) and weak or negative (hyperreactive) TGF- $\beta$ (E-F) and HLA-DR (G-H) expression (arrows) mainly in macrophages adjacent to productive female worms and an attached HLA-DR ${ }^{+}$giant cell (*). Immune cells further distant from the worm are HLA-DR ${ }^{+}$(arrowhead in $\mathrm{H}$ ), whereas they are TGF- $\beta$-positive (F) and macrophages adjacent to the worm are HLA-DR-negative $(\mathrm{H})$. (J-M) Moribund or dead $\mathrm{mf}$ in a nodular microgranuloma being attacked by macrophages strongly (hyporeactive) or faintly (hyperreactive) expressing TGF- $\beta$ or HLA-DR (arrows). $(\mathrm{K}+\mathrm{M})$ are serial sections. $(\mathrm{N}-\mathrm{Q})$ Mainly $\mathrm{CD}^{+} \mathrm{T}$ lymphocytes express $\mathrm{TGF}-\beta$ strongly in a nodule from a hyporeactive and weakly in a nodule from a hyperreactive patient. $(\mathrm{N}+\mathrm{P}$ and $\mathrm{O}+\mathrm{Q})$ are serial sections.

$(\mathrm{P}+\mathrm{Q}) \mathrm{CD} 3$-staining. Scale bars $=50 \mu \mathrm{m}$.

was derived from a German boy, who became infected in Cameroon, developed hyperreactive dermatitis and was treated with 2 standard doses of $0.15 \mathrm{mg} / \mathrm{kg}$ IVM 4 and 6 months prior to nodulectomy. Only a subgroup of these nodules was stained with different Abs depending on fixation and needed information.
The patients in this study were HIV-negative. The HIV infection was excluded by testing or, in Liberia, Mali, Burkina Faso, and Yemen by epidemiology, since nodulectomy had taken place before the onset of the HIV1 epidemic in the investigated onchocerciasis foci. 
Table 1. Untreated and ivermectin-treated hypo- and hyperreactive onchocerciasis patients and numbers of examined onchocercomas (nodules), lymph nodes and skin biopsies

\begin{tabular}{|c|c|c|c|c|c|c|}
\hline $\begin{array}{l}\text { Form of } \\
\text { onchocerciasis, } \\
\text { no. of patients }\end{array}$ & $\begin{array}{l}\text { Ivermectin } \\
\text { treatment } \\
\text { dose }\end{array}$ & $\begin{array}{l}\text { No. of } \\
\text { nodules } \\
\left(\text { TGF- } \beta^{\text {a }}\right)\end{array}$ & $\begin{array}{l}\text { No. of } \\
\text { lymph } \\
\text { nodes/ } \\
\text { patients }\end{array}$ & $\begin{array}{l}\text { No. of } \\
\text { skin } \\
\text { biopsies/ } \\
\text { patients }\end{array}$ & $\begin{array}{l}\text { Endemic } \\
\text { country }\end{array}$ & Reference \\
\hline \multicolumn{7}{|l|}{ Hyperreactive } \\
\hline 47 & None & $61(46)$ & 4 & 17 & Liberia & (Albiez et al. 1985) \\
\hline 3 & & $3(3)$ & 2 & 0 & Yemen & (Büttner et al. 1982) \\
\hline 1 & $2 \times 0.15 \mathrm{mg} / \mathrm{kg}$ & $1(0)$ & 0 & 0 & Cameroon & (Korten et al. 1998) \\
\hline 2 & $1 \times 0.15 \mathrm{mg} / \mathrm{kg}$ & $2(2)$ & 1 & 0 & Liberia & (Darge and Büttner, 1995) \\
\hline \multicolumn{7}{|l|}{ Hyporeactive } \\
\hline 25 & None & $35(13)$ & 1 & 2 & Ghana & (Hoerauf et al. 2003) \\
\hline 30 & & $42(32)$ & 3 & 20 & Liberia & (Albiez et al. 1988b) \\
\hline 12 & & $17(17)$ & 0 & 0 & Burkina $\mathrm{F}^{\mathrm{b}}$ & (Büttner et al. 1988) \\
\hline 13 & & $16(16)$ & 0 & 0 & Mali & (Büttner et al. 1988) \\
\hline 14 & & $20(4)$ & 0 & 0 & Uganda & (Fischer et al. 1993) \\
\hline 1 & & 0 & 1 & 0 & Yemen & (Büttner et al. 1982) \\
\hline 9 & $1-3 \times 0 \cdot 15 \mathrm{mg} / \mathrm{kg}$ & $12(6)$ & 0 & 0 & Uganda & (Fischer et al. 1993) \\
\hline 2 & $1 \times 0 \cdot 15 \mathrm{mg} / \mathrm{kg}$ & $2(2)$ & 4 & 0 & Liberia & (Albiez et al. 1988b) \\
\hline
\end{tabular}

a 49 nodules from 50 untreated hyperreactive patients and 82 nodules from 82 untreated hyporeactive patients were examined for TGF- $\beta$, the other nodules for other markers as outlined in the Materials and methods section;

b Burkina Faso.

The Ethics Commission of the Medical Board, Hamburg, had approved nodulectomies for research purposes. Nodulectomies were also, generally, supported by the World Health Organization (WHO, 1987). The design of the different drug studies had been approved by the authorities and by ethic committees of the respective African countries (see references). The procedures used were in accordance with the Declaration of Helsinki (1975, and its revisions in 1983, 2000 and 2002, where applicable). The nodulectomies were performed under aseptic conditions and local anaesthesia as described (Albiez et al. 1988a). Among the onchocercomas excised by us, and those that were sent to us by other groups were usually up to $5 \%$ lymph nodes, which had been misdiagnosed as onchocercomas (Albiez et al. 1988a). In nodule series from patients after long IVM mass treatment, vector control and those with hyperreactive infection the percentages were higher because these patients have fewer onchocercomas. In total, 16 lymph nodes from 7 hyperreactive and 9 hyporeactive patients were included in this study, 11 from untreated and 5 from IVM-treated patients (for references see Table 1). From 22 untreated hyporeactive and 17 untreated hyperreactive patients, skin samples were taken from the wound edge adjacent to the excised onchocercomas (Table 1).

\section{Immunohistochemistry}

Onchocercomas, lymph nodes and skin were fixed in $4 \%$ buffered formaldehyde or in $80 \%$ ethanol and embedded in paraffin using standard methods. The number, sex, productivity, and vitality of the worms were determined morphologically (Korten et al.
2008). As a rich cellular infiltrate is dependent on the presence of at least one mf-producing female worm or on dead worms (Wildenburg et al. 1998; Korten et al. 2008), and host TGF- $\beta$ expression is induced by nulliparous, previously or presently productive or dead female worms in hyporeactive onchocercomas (Korten et al. 2010), nodules with at least one such female worm were used for this immunological comparison between the two forms of onchocerciasis. Young nulliparous female worms were present in larger nodules from hyporeactive patients. Male worms were present in many nodules with females; in this nodule series males did not occur alone.

For staining of latent TGF- $\beta 1$, only formaldehydefixed nodules could be used because the antiserum did not stain ethanol-fixed host tissues (albeit ethanol-fixed worm tissue, Korten et al. 2009). Therefore not all nodules examined could be stained with anti-TGF- $\beta$ serum. The sections were pretreated with an endogenous peroxidase-blocking agent as part of the anti-goat HRP-AEC Cell \& Tissue Staining Kit (horseradish peroxidase-3amino-9-ethyl-carbazole; R\&D Systems, Wiesbaden, Germany). As described previously (Korten et al. 2009 , 2010) latent TGF- $\beta 1$ was labelled with a specific polyclonal IgG antibody produced with recombinant goat anti-human latency peptide/ protein (LAP; R\&D Systems), diluted 1:200 and for non-filarial samples 1:10 in 1\% FCS/TBS. Titration experiments for optimal dilutions of the primary antibody and positive control staining were performed on known $\mathrm{LAP}^{+}$prostate carcinoma samples (Steiner et al. 1994). Non-specific goat serum with the same $\operatorname{IgG}$ concentration was applied for negative 
controls. Pre-diluted biotinylated anti-goat mAb served as secondary antibody. For visualization, slides were incubated with streptavidin-HRP and developed with the brown chromogenic substrate AEC or with the streptavidin-biotin Super Sensitive Detection Kit (BioGenex, San Ramon, CA, USA) and Vector Red Substrate Kit (Vector Labs, Burlingame, CA, USA). When an affinity-purified goat antihuman LAP antibody and a mouse monoclonal antibody $(\mathrm{mAb})$ against TGF- $\beta$ became available, a number of sections were stained to confirm the specificity of the labelling using the first antibodies (not shown).

TGF- $\beta$-positive or -negative cell types were determined morphologically and on the basis of a similar location of cells stained with the following differentiation markers on serial sections. Mainly mAbs were applied (DakoCytomation, Hamburg, Germany): anti-CD68 (clone PG-M1) for mature macrophages, anti-L1 protein (synonymous with clone MAC387) for immature macrophages, antiMHC class II molecule HLA-DR (clone TAL. 1B5), and anti-mast cell tryptase (clone AA1); polyclonal antibodies against CD3 to detect all T lymphocytes, and against $\operatorname{IgE}, \operatorname{IgG} 1$ and $\operatorname{IgG} 4$. For these antibodies the APAAP technique was applied according to the protocol from DakoCytomation, using secondary rabbit anti-mouse and mouse anti-rabbit Abs and Fast Red TR salt (Sigma, Deisenhofen, Germany). Haematoxylin (Merck, Darmstadt, Germany) served as counter stain.

Not all nodules could be stained with all antibodies. Some nodules had only been stained with the differentiation markers mentioned above and with others specific for eosinophils, neutrophils, alpha 1-antichymotrypsin, and prostaglandin $\mathrm{E}_{2}$, which are not mentioned here. Forty-nine nodules from hyperreactive and 82 nodules from hyporeactive patients were stained for TGF- $\beta$ and a subgroup of these also for the above-mentioned differentiation markers using consecutive sections.

\section{Statistical analysis}

For the analysis of difference between nodules from hyperreactive patients with low intensity of TGF- $\beta$ staining and nodules from hyporeactive patients with strong TGF- $\beta$ staining the chi square test was applied (http://math.hws.edu/javamath/ryan/ ChiSquare.html), assuming $P<0.05$ to be a significant difference.

\section{RESULTS}

\section{Hyperreactive patients show reduced TGF- $\beta$ expression}

Onchocercomas with productive or previously productive older worms revealed a much stronger expression of TGF- $\beta 1$ (denoted as TGF- $\beta$ hereafter) and more frequently positive cells around and between sections of the adult worms, when patients had the hyporeactive form of onchocerciasis (Fig. 1A, C and $\mathrm{E}$ ) in contrast to the hyperreactive form (Fig. 1B, $\mathrm{D}$ and $\mathrm{F}$ ). This was observed in numerous mature $\mathrm{CD}^{+} 8^{+}$and L1 protein ${ }^{+}$(data not shown, see Discussion section) macrophages forming a wall adjacent to the cuticle of the adult worms in both polar forms (Fig. $1 \mathrm{~A}-\mathrm{F}$ ). Few TGF- $\beta^{+}$macrophages and numerous TGF $-\beta^{+}$lymphocytes occurred more distant from the adult worms in the nodule centre, but again with reduced expression levels in hyperreactive nodules (Fig. 1F). Similarly, macrophages attacking dead $\mathrm{mf}$ expressed more TGF- $\beta$ in nodules from hyporeactive (Fig. 1J) than in those from hyperreactive patients (Fig. 1K). $\mathrm{CD}^{+} \mathrm{T}$ cells (Fig. 1P and $\mathrm{Q}$ ) in perivascular infiltrates strongly expressed TGF- $\beta$ in hyporeactive (Fig. $1 \mathrm{~N}$ ), but very weakly and less frequently in hyperreactive onchocercomas (Fig. 1O). Mast cells and fibrocytes were also weaker TGF- $\beta^{+}$in hyperreactive onchocercomas (data not shown).

The difference in TGF- $\beta$ expression was always obvious. In hyporeactive nodules, the stained immune cells were mainly strongly or moderately TGF- $\beta$ positive. In hyperreactive nodules the staining of the macrophages varied mainly from negative to weakly positive, seldom to moderately positive, but the cells were never as strongly stained as the positive cells in hyporeactive nodules. Figures 1-3 clearly demonstrate the different intensities in the two groups of patients. These figures are representative; they were not selected for the intensity of TGF- $\beta$ staining. The difference between the 49 hyperreactive and the 82 hyporeactive TGF- $\beta$ stained nodules is significant, when comparing the weak and strong expression for the total nodule numbers between the two groups $(P<0 \cdot 001$, chi square test).

A quantification and statistical analysis of the frequency of TGF- $\beta$ stained cells appeared not to be adequate since most nodules from hyperreactive patients contained many more immune cells than those from hyporeactive patients (Büttner and Racz, 1983) and weighed between $1 \cdot 7$ and $8 \cdot 3 \mathrm{~g}$ in contrast to most hyporeactive nodules weighing less than $1 \cdot 3 \mathrm{~g}$ (Albiez et al. 1988a). Despite this high number of immune cells elicited by only one or two female worms in hyperreactive nodules, most of these immune cells were only weakly or not TGF- $\beta$ stained. This difference in cellular infiltration was also evident between the large lymph nodes of hyperreactive patients and the fibrotic nodes from hyporeactive patients (Connor et al. 1983; Racz et al. 1983) and between the onchodermatitis skin from hyperreactive patients and the often clinically only scarcely altered skin in the hyporeactive group (Connor et al. 1983). 

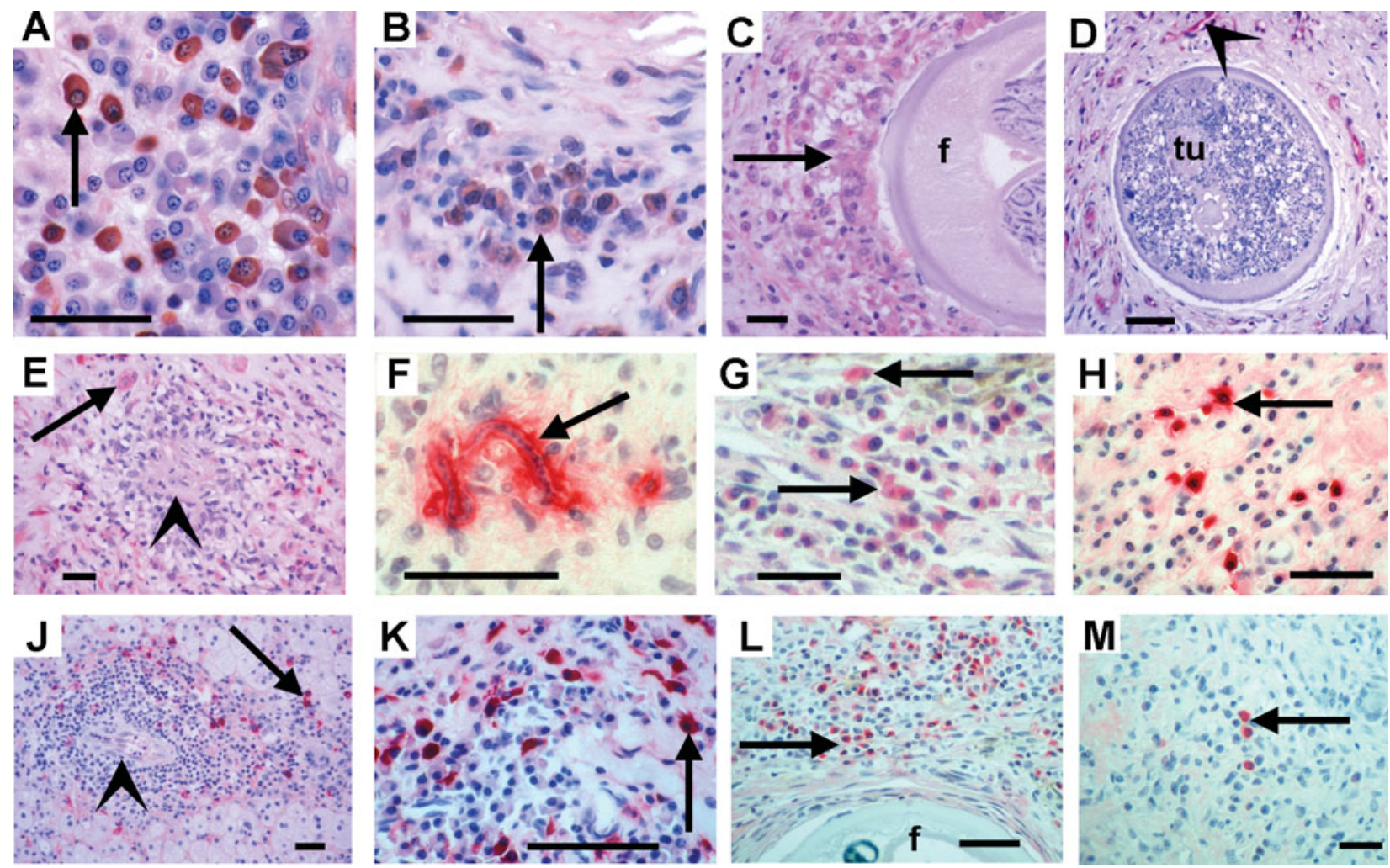

Fig. 2. Immunohistological comparison of TGF- $\beta$ and immunoglobulin production by plasma cells and macrophages in onchocercomas from hypo- and hyperreactive patients in Liberia and Uganda. (A-B) Plasma cells (arrow) strongly (hyporeactive) and faintly (hyperreactive) expressing TGF- $\beta$. Untreated patients. (C) Weakly TGF- $\beta^{+}$macrophages (arrows) attached to a productive female worm (f) 2 months after IVM. Hyperreactive. (D) Only TGF- $\beta^{+}$endothelia (arrowhead) are seen but nearly no immune cells near to a moribund female worm harbouring a pleomorphic neoplasm (tu) filling the entire pseudocoeloma cavity (Duke et al. 2002; Brattig et al. 2010). Hyperreactive. (E) Weakly TGF- $\beta^{+}$ macrophages (arrow) around a microgranuloma (arrowhead) with a dead $\mathrm{mf}$ in a nodule from a hyperreactive patient 2 months after IVM treatment. (F) A dead mf coated by IgG1 (arrow) in a nodule from a hyperreactive German child after IVM. (G-H) Weakly (hyporeactive, $48 \mathrm{~h}$ after IVM) and strongly (hyperreactive, untreated) IgG1 producing plasma cells (arrows). TGF- $\beta$ (J-K) or HLA-DR (L-M) (arrows) faintly (hyporeactive) and strongly (hyperreactive) IgE producing plasma cells (arrows) in perivascular infiltrates. Untreated patients. (L-M) Many (hyporeactive) and few (hyperreactive) IgG4 producing plasma cells (arrows) in nodules with productive female worms (f); $2-3$ days after IVM treatment. Scale bars $=20 \mu \mathrm{m}(\mathrm{A}, \mathrm{B}, \mathrm{E}) ; 50 \mu \mathrm{m}(\mathrm{C}-\mathrm{D}, \mathrm{F}-\mathrm{M})$.

Furthermore, the proportions of TGF- $\beta$ negative or positive young L1 protein positive macrophages and TGF- $\beta$ negative prostaglandin E2 expressing macrophages varied between the two groups, and in hyporeactive nodules strongly TGF- $\beta^{+}$cells were so abundant and often densely packed, that it would have been difficult to quantify them in contrast to clear-cut populations of FOXP3 + lymphocytes (Korten et al. 2008).

\section{Stronger HLA-DR expression in hyporeactive than in hyperreactive patients}

Since all antigen-specific $\mathrm{CD} 4^{+} \mathrm{T}$ cells, including $\mathrm{T}$ helper and Treg cells, are induced and activated via MHC class II molecules such as HLA-DR on antigen-presenting cells, we compared HLA-DR expression in relation to TGF- $\beta$ between the two forms of onchocerciasis. HLA-DR expression was equally strong in both forms, but HLA-DR ${ }^{+}$cells occurred more abundantly in hyporeactive nodules (Fig. $1 \mathrm{G}$ and $\mathrm{H}$ ). This was particularly striking for the completely HLA-DR-negative macrophages in the cell walls adjacent to the cuticle of productive females in hyperreactive nodules (Fig. 1H) in contrast to the homogenously HLA-DR ${ }^{+}$macrophages adjacent to females in hyporeactive onchocercomas (Fig. 1G). HLA-DR ${ }^{+}$macrophages and lymphocytes infiltrated the fibrous tissue at some distance from the adult worms in hypo- and hyperreactive nodules (Fig. 1H). But the expression was stronger in hyporeactive nodules. Central and subcapsular infiltrates were densely packed with strongly HLA-DR ${ }^{+}$cells. In contrast to the cells adjacent to adult worms, strongly HLA-DR expressing macrophages closely 

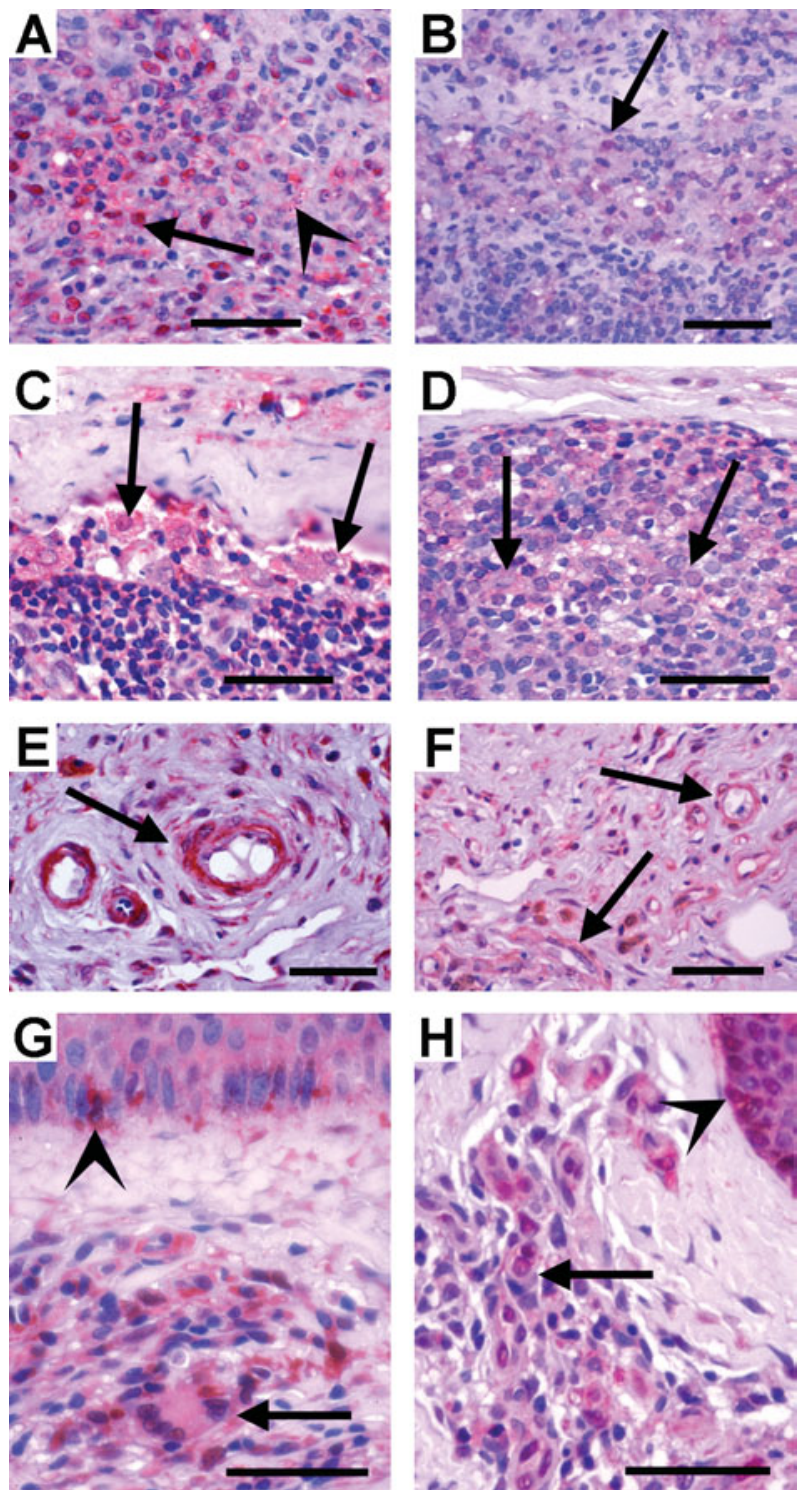

Fig. 3. Immunohistological comparison of TGF- $\beta$ expression in lymph nodes (A-F) and skin $(\mathrm{G}-\mathrm{H})$ from hypo- and hyperreactive patients in Liberia and Uganda. (A-B) Strong (hyporeactive) and faint TGF- $\beta$ expression (hyperreactive) in lymphocytes (arrows) adjacent to remnants of nearly resorbed $\mathrm{mf}$ (arrowhead). Untreated patients. (C-D) Strong (hyporeactive) and faint TGF- $\beta$ expression (hyperreactive) in macrophages (arrows); 1 day or 5 weeks after IVM treatment. (E-F) Strongly

(hyporeactive) TGF- $\beta^{+}$and faintly (hyperreactive) TGF- $\beta$ expressing endothelia of blood vessels; 1 day or 5 weeks after IVM treatment. (G-H) Skin from untreated patients. (G) Macrophages and lymphocytes with varying degrees of TGF $-\beta$ expression (arrow) accumulating in a dermal infiltrate and strongly TGF- $\beta^{+}$Langerhans cells in the epidermis (arrowhead; hyporeactive). (H) A similar accumulation of less strongly TGF- $\beta$ expressing cells in a dermal infiltrate and of Langerhans cells in the epidermis (arrowhead) of a hyperreactive patient. Scale bars $=50 \mu \mathrm{m}$.

encircled degenerated $\mathrm{mf}$ in both forms, but accumulated in higher numbers in the hyporeactive infection (Fig. 1L and M).
In summary, these results show that TGF- $\beta$ expression positively correlates with HLA-DR expression, particularly in the cellular reaction around adult worms and less around $\mathrm{mf}$.

\section{Plasma cells expressed more IgE and IgG1, but less IgG4 in hyperreactive patients}

$\mathrm{CD}_{20}{ }^{+} \mathrm{B}$ cells, including plasma cells, were abundantly found in all onchocercomas with $\mathrm{mf}$ producing and/or degenerating adult worms. Plasma cells often accumulated in lymphocyte infiltrates and produced more TGF- $\beta$ in hyporeactive (Fig. 2A) than in hyperreactive onchocercomas (Fig. 2B). Many plasma cells produced IgE or IgG1 in both polar forms, but strongly IgG1 or IgE expressing plasma cells occurred more frequently in hyperreactive (Fig. 2F, H, K) than in hyporeactive nodules (Fig. 2G and J). Inversely, IgG4 was abundantly expressed in hyporeactive (Fig. 2L) and scarcely in hyperreactive nodules (Fig. 2M).

In summary, weak TGF- $\beta$ and IgG4 expression by plasma cells correlated with hyperreactivity, inversely to $\mathrm{IgE}$ and $\mathrm{IgG} 1$.

\section{TGF- $\beta$ in nodules from IVM-treated patients}

After IVM treatment the macrophages attached to the female worms did not or only weakly expressed TGF- $\beta$ in hyperreactive patients (Fig. 2C). In hyperreactive patients, this weak TGF- $\beta$ expression was also seen around dead or moribund adult worms such as those with neoplasms (Fig. 2D). Degenerated $\mathrm{mf}$ were encircled by macrophages, eosinophils, some $\mathrm{CD}^{+} \mathrm{T}$ lymphocytes and mast cells forming microgranulomas in nodules from IVM-treated hyperreactive patients, and these cells were also only weakly TGF- $\beta$ positive (Fig. $2 \mathrm{E}$ ).

\section{Hyperreactivity imparts reduced TGF- $\beta$ expression in lymph nodes and skin}

In contrast to stronger expression in hyporeactive patients (Fig. 3A and C) many lymphocytes (Fig. 3B) and macrophages (Fig. 3D) showed less TGF- $\beta$ in lymph nodes of hyperreactive patients. Likewise, numerous endothelia of small blood vessels in lymph nodes were TGF $-\beta^{+}$in both polar forms, but they were less strongly stained in hyperreactive patients (Fig. 3E and F). Furthermore, TGF- $\beta$ expression was observed in numerous cells in small inflammatory infiltrates in the dermis as well as in Langerhans cells in the basal layer of the epidermis (Fig. 3G and $\mathrm{H}$ ). The expression was higher in the skin from hyporeactive than in skin from hyperreactive patients (Fig. 3G and $\mathrm{H}$ ). But the difference appeared to be less than observed in onchocercomas and lymph nodes from hyporeactive patients. 
In summary, a similar weak TGF- $\beta$ expression occurred in lymph nodes and skin as in onchocercomas from hyperreactive patients.

\section{DISCUSSION}

Comparing the in situ expression of latent TGF- $\beta$ in onchocercomas, lymph nodes and skin between hyperreactive and hyporeactive patients, we show that all three organs from hyperreactive patients expressed lower levels of TGF- $\beta$ and reduced numbers of TGF- $\beta^{+}$cells despite a strong inflammatory infiltrate. As TGF- $\beta$ was less intensely and less frequently expressed but present in hyperreactive nodules, this indicates the ability of these patients to express TGF- $\beta$. It might reflect that TGF- $\beta$ expression is up-regulated and latent TGF- $\beta$ (LAP) released also in hyperreactive patients, but more quickly cleaved into active TGF- $\beta$, e.g. by proteases such as metalloproteinases (Taylor, 2009; Korten et al. 2010). Alternatively, LAP could bind to cellsurface proteins such as integrins undergoing a conformational change and releasing active TGF- $\beta$, which was not detected by the TGF- $\beta$ antibody used. An argument against this is that active TGF- $\beta$ binding to receptors of effector cells should lead to suppression of hyperreactivity, which is not the case. Another explanation might be that cellular production could reach a point of exhaustion in hyperreactivity in response to a strong and constant inflammation overwhelming any regulatory effect.

The hyperreactive response in primary infections of all non-endemic and endemic infected individuals indicates that any initial TGF- $\beta$ response to inflammation is too weak to suppress and needs further signals to develop. We presume that filarial and not endobacterial antigens provide such signals, since depletion of Wolbachia did not alter TGF- $\beta$ expression (Korten et al. 2010). Furthermore, only in hyporeactive patients did filarial antigens from adult, predominantly female, worms elicit a substantial TGF- $\beta$ expression, which rises with the first $\mathrm{mf}$ production and is sustained in non-productive phases and during worm degeneration, death and resorption (Korten et al. 2010). This local TGF- $\beta$ production increases with worm burden, suppressing defensive Th2 responses and counterbalancing immunopathology. Since TGF- $\beta$ induces $\mathrm{CD}^{+}{ }^{+}$Treg cells, a growing worm load promotes the generation of $O$. volvulus-specific as well as non-specific Treg cells found in onchocercomas (Hoerauf et al. 2005; Korten et al. 2008).

One explanation for the low TGF- $\beta$ expression in hyperreactive tissues could be that chronically infected hyperreactive patients cannot mount a sufficient TGF- $\beta$ response during the hyperreactive phase due to a pre-disposing genetic factor. The Ov1 locus on chromosome $2 \mathrm{p}$ is linked to $\mathrm{mf}$ loads in the skin and resistance in onchocerciasis, close to a region for asthma, also a Th2-driven condition (Timmann et al. 2008). Moreover, since protein kinase $\mathrm{C}$ epsilon is a functional candidate gene of Ov1 ('Timmann et al. 2008) and protein kinase $C$ epsilon mediates increased TGF- $\beta$ production in mice (Koyanagi et al. 2007) this locus might be associated with the observed differences in TGF- $\beta 1$ expression. Therefore, hyperreactive patients may have developed and sustained a strong Th2 response from the beginning of infection that quickly killed and removed $\mathrm{mf}$. We assume that this prohibited the development of TGF- $\beta$-mediated tolerance in hypoendemic regions. However, in meso- and hyperendemic areas, tolerance eventually overcomes hyperreactivity due to higher transmission pressure and the increasing worm load. In onchocercomas from untreated and IVM-treated hyperreactive patients, degenerated $\mathrm{mf}$ were encircled by macrophages, eosinophils, $\mathrm{CD}^{+} \mathrm{T}$ cells and some mast cells (Korten et al. 1998), but these cells were TGF$\beta$-negative or faintly TGF- $\beta^{+}$in contrast to hyporeactive nodules (for the hyporeactive form only, see Korten et al. 2010). This and the similarly weak TGF- $\beta$ reaction around dead adult worms showed the independence of the low TGF- $\beta$ expression from the worm status, reinforcing the concept of predisposition. This is also supported by a study showing that TGF- $\beta$ release into the circulation is genetically controlled and correlates with the occurrence and severity of certain diseases (Grainger et al. 1999). In addition, hyperreactive patients from both Ghana and Guinea were shown to have a predisposition for increased IL-13 signalling and thus, stronger Th2 responses (Hoerauf et al. 2005). Since TGF- $\beta$ suppresses Th1/2 cell proliferation and differentiation ( $\mathrm{Li}$ et al. 2006), the lack of TGF- $\beta$ could promote local and systemic cell proliferation, which is increased in patients with severe and chronic skin pathology (Timmann et al. 2003).

TGF- $\beta$ not only suppresses immune cells, but can also promote fibrous tissue generation and fibrosis. The much stronger fibrous tissue reaction around one or two female worms in the presence of scarce TGF- $\beta$ in hyperreactive nodules may be an argument against such a role of TGF- $\beta$ in hyperreactive patients. However, the increased fibrosis seen in lymph nodes and skin of older hyporeactive patients with longstanding infections could partially result from their high TGF- $\beta$ production apart from immune complexes of $O$. volvulus $\mathrm{mf}$ antigens, which cause inflammation and formation of additional laminae of vascular walls and perivascular fibrosis (Connor et al. 1983). Since we observed TGF- $\beta^{+}$macrophages and lymphocytes in the hyporeactive lymph nodes, such TGF- $\beta$ expression could reflect suppressive macrophage populations in the regional lymph nodes. In line with this, a spreading of alternatively activated macrophages from the site of infection to the regional lymph nodes with the onset of $\mathrm{mf}$ release 
is observed in murine filarial infection (Taylor et al. 2006). Brattig et al. (2009) reported a lower production of prostaglandin $\mathrm{E}_{2}$ and TGF- $\beta$ by macrophages in onchocercomas from hyperreactive than in those from hyporeactive patients, and the production of these two compounds in different suppressive macrophage subtypes.

Plasma cells were a major source of TGF- $\beta$ apart from macrophages, $\mathrm{T}$ cells, mast cells, endothelial cells, and fibrocytes in hyporeactive (Korten et al. 2010) and hyperreactive onchocercomas, but the expression was less in the hyperreactive form. The enhanced $\mathrm{IgE}$ and $\mathrm{IgG} 1$, but scarce $\mathrm{IgG} 4$ expression by plasma cells in hyperreactive onchocercomas is in line with reports on the inverse role of these immunoglobulins (Brattig et al. 1994; Ottesen, 1995; Brattig, 2004). The production of $\mathrm{IgG} 4$, a predominantly blocking, anti-inflammatory and tolerance-inducing antibody (van der Neut Kolfschoten et al. 2007; Aalberse et al. 2009) by B cells is induced by IL-10 and TGF- $\beta$ producing Tr1 and natural Treg cells in onchocerciasis (Satoguina et al. 2008). Therefore, our finding of simultaneously high TGF- $\beta$ and IgG4 expression by plasma cells in hyporeactive infections is concordant with this notion. This supports the assumption of parallel expression by several plasma cells or possibly the same plasma cells being regulatory B cells (Lund, 2008).

Brattig et al. (1987) showed increased HLA-DR expression by peripheral blood leukocytes in hyperreactive patients. In contrast, we found substantial numbers of positive cells, but decreased local expression intensities in hyperreactive onchocercomas compared to strong and more abundant expression in hyporeactive ones. This might indicate a local exhaustion of expression in chronic hyperreactivity. However, since HLA-DR molecules induce and activate antigen-specific $\mathrm{CD}^{+}{ }^{+} \mathrm{T}$ cells in general, and can be expressed by activated Treg cells (Muixí et al. 2008; Battaglia and Roncarolo, 2009), the strong expression in hyporeactive patients could also lead to the generation of cognate $\mathrm{CD}^{+}$Treg cells and not only of $\mathrm{CD}^{+}$helper cells. An association of HLA-DR molecules with the clinical spectrum of onchocerciasis has been reported by Meyer et al. (1994). A clear association exists between HLA-DR expression and autoimmune diseases such as rheumatoid arthritis and thyroid autoimmune disease (Muixí et al. 2008). In both, the complex of the presented peptide with the MHC class II molecule HLA-DR can be recognized by cognate effector and regulatory $\mathrm{T}$ cells promoting either autoreactivity or tolerance.

In conclusion, this study showed an association between chronic Th2-mediated hyperreactivity in onchocerciasis and reduced local expression of the suppressive cytokine TGF- $\beta$ by various cell types, primarily macrophages, $\mathrm{T}$ cells and plasma cells. This low TGF- $\beta$ expression was found in all infection sites irrespective of the worm status or the presence of $\mathrm{mf}$. It was further linked with reduced HLA-DR expression. Therefore, we suggest a new tolerance-promoting role for MHC class II-mediated immune responses in conjunction with local TGF- $\beta$ expression, which develops in hyporeactive, but not in hyperreactive patients. This might be important not only for the understanding of hyperreactivity to filarial nematodes, but also for other Th2-mediated diseases such as allergy and asthma.

\section{ACKNOWLEDGEMENTS}

We thank Ingeborg Albrecht, Marlis Badusche, Kerstin Fischer, and Frank Geisinger for technical assistance. $\mathrm{LAP}^{+}$prostate carcinoma samples were kindly provided by Andreas Erbersdobler (Institute for Pathology, University Medical Centre Hamburg-Eppendorf).

\section{F INANCIAL SUPPORT}

This study was supported by the EU project 'Sustainable Control of Onchocerciasis Today and Tomorrow SCOOTT' (contract-no. 032321) and the Volkswagen Stiftung (project no. I/84 159).

\section{REFERENCES}

Aalberse, R. C., Stape1, S. O., Schuurman, J. and Rispens, T. (2009). Immunoglobulin G4: an odd antibody. Clinical and Experimental Allergy 39, 469-477.

Albiez, E. J., Büttner, D. W. and Duke, B. O. (1988a). Diagnosis and extirpation of nodules in human onchocerciasis. Tropical Medicine and Parasitology 39, 331-346.

Albiez, E. J., Gallin, M., Erttmann, K. D., Racz, P. and Büttner, D. W. (1985). Characteristics of chronic severe onchodermatitis in Liberia. Fournal of the Liberian Medical and Dental Association 15, 120-125.

Albiez, E. J., Walter, G., Kaiser, A., Ranque, P., Newland, H. S., White, A. T., Greene, B. M., Taylor, H. R. and Büttner, D. W. (1988b). Histological examination of onchocercomata after therapy with ivermectin. Tropical Medicine and Parasitology 39, 93-99.

Battaglia, M. and Roncarolo, M. G. (2009). The fate of human Treg cells. Immunity 30, 763-765.

Brattig, N. W. (2004). Pathogenesis and host responses in human onchocerciasis: impact of Onchocerca filariae and Wolbachia endobacteria. Microbes and Infection $\mathbf{6}$, 113-128.

Brattig, N. W., Hoerauf, A., Fischer, P. U., Liebau, E., Bandi, C., Debrah, A., Büttner, M. and

Büttner, D. W. (2010). Immunohistological studies on neoplasms of female and male Onchocerca volvulus: filarial origin and absence of Wolbachia from tumor cells. Parasitology 137, 841-854.

Brattig, N. W., Krawitz, I., Abakar, A. Z., Erttmann, K. D., Kruppa, T. F. and Massougbodji, A. (1994). Strong IgG isotypic antibody response in sowdah type onchocerciasis. The Fournal of Infectious Diseases 170, 955-961. 
Brattig, N. W., Schwohl, A., Hoerauf, A. and Büttner, D. W. (2009). Identification of the lipid mediator prostaglandin $\mathrm{E}_{2}$ in tissue immune cells of humans infected with the filaria Onchocerca volvulus. Acta Tropica 112, 231-235.

Brattig, N. W., Tischendorf, F. W., Albiez, E. J., Büttner, D. W. and Berger, J. (1987). Distribution pattern of peripheral lymphocyte subsets in localized and generalized form of onchocerciasis. Clinical Immunology and Immunopathology 44, 149-159.

Büttner, D. W. and Racz, P. (1983). Macro- and microfilariae in nodules from onchocerciasis patients in the Yemen Arab Republic. Tropenmedizin und Parasitologie 34, 112-121.

Büttner, D. W., Albiez, E. J., Essen, J. von and Erichsen, J. (1988). Histological examination of adult Onchocerca volvulus and comparison with the collagenase technique. Tropical Medicine and Parasitology 39, 390-417.

Büttner, D. W., Laer, G. von, Mannweiler, E. and Büttner, M. (1982). Clinical, parasitological and serological studies on onchocerciasis in the Yemen Arab Republic. Tropenmedizin und Parasitologie 33, 201-212.

Connor, D. H., Gibson, D. W., Neafie, R. C., Merighi, B. and Buck, A. A. (1983).

Sowda-onchocerciasis in north Yemen: a clinicopathologic study of 18 patients. American Fournal of Tropical Medicine and Hygiene 32, 123-137.

Cooper, P. J., Mancero, T., Espinel, M., Sandoval, C., Lovato, R., Guderian, R. H. and Nutman, T. B. (2001). Early human infection with Onchocerca volvulus is associated with an enhanced parasite-specific cellular immune response. Fournal of Infectious Diseases 183, 1662-1668.

Darge, K. and Büttner, D. W. (1995). Ivermectin treatment of hyperreactive onchodermatitis (sowda) in Liberia. Tropical Medicine and Parasitology 46, 206-212.

Doetze, A., Satoguina, J., Burchard, G., Rau, T., Löliger, C., Fleischer, B. and Hoerauf, A. (2000). Antigen-specific cellular hyporesponsiveness in a chronic human helminth infection is mediated by $\mathrm{T}(\mathrm{h}) 3 / \mathrm{T}(\mathrm{r}) 1$ type cytokines IL-10 and transforming growth factorbeta but not by a $\mathrm{T}(\mathrm{h}) 1$ to $\mathrm{T}(\mathrm{h}) 2$ shift. International Immunology 12, 623-630.

Duke, B. O. L., Marty, A. M., Peet, D. L., Pardon, J., Pion, S. D. S., Kamgno, J. and Boussinesq, M. (2002). Neoplastic change in Onchocerca volvulus and its relation to ivermectin treatment. Parasitology 125, 431-444.

Fischer, P., Kipp, W., Bamuhiga, J., Binta-Kawa, J., Kiefer, A. and Büttner, D. W. (1993). Parasitological and clinical characterization of Simulium neaveitransmitted onchocerciasis in western Uganda. Tropical Medicine and Parasitology 44, 311-321.

Gallin, M. (1987). Clinical and parasitological studies on the chronic hyperreactive onchodermatitis in Liberia. [In German] Dissertation, Faculty of Medicine, University of Hamburg, Germany.

Grainger, D. J., Heathcote, K., Chiano, M., Snieder, H., Kemp, P. R., Metcalfe, J. C., Carter, N. D. and Spector, T. D. (1999). Genetic control of the circulating concentration of transforming growth factor type beta1. Human Molecular Genetics 8 , 93-97.
Hoerauf, A., Kruse, S., Brattig, N. W., Heinzmann, A., Mueller-Myhsok, B. and Deichmann, K. A. (2002). The variant Arg110Gln of human IL-13 is associated with an immunologically hyper-reactive form of onchocerciasis (sowda). Microbes and Infection 4, $37-42$.

Hoerauf, A., Mand, S., Volkmann, M., Büttner, M., Marfo-Debrekyei, Y., Taylor, M., Adjei, O. and Büttner, D. W. (2003). Doxycycline in the treatment of human onchocerciasis: kinetics of Wolbachia endobacteria reduction and of inhibition of embryogenesis in female Onchocerca worms. Microbes and Infection 5, 261-273.

Hoerauf, A., Satoguina, J., Saeftel, M. and Specht, S. (2005). Immunomodulation by filarial nematodes. Parasite Immunology 27, 417-429.

Kilian, H. D. (1988). The use of a topical Mazzotti test in the diagnosis of onchocerciasis. Tropical Medicine and Parasitology 39, 235-238.

Kilian, H. D. and Nielsen, G. (1989). Cell-mediated and humoral immune responses to BCG and rubella vaccinations and to recall antigens in onchocerciasis patients. Tropical Medicine and Parasitology 40, 445-453.

King, C. L. and Nutman, T. B. (1991). Regulation of the immune response in lymphatic filariasis and onchocerciasis. Immunology Today 12, A54-A58.

Korten, S., Badusche, M., Büttner, D. W., Hoerauf, A., Brattig, N. and Fleischer, B. (2008). Natural death of adult Onchocerca volvulus and filaricidal effects of doxycycline induce local FOXP3 + /CD4 + regulatory $\mathrm{T}$ cells and granzyme expression. Microbes and Infection 10, 313-324.

Korten, S., Büttner, D. W., Schmetz, C., Hoerauf, A., Mand, S. and Brattig, N. (2009). The nematode parasite Onchocerca volvulus generates the transforming growth factor-beta (TGF-beta). Parasitology Research 105, 731-741.

Korten, S., Kaifi, J. T., Büttner, D. W. and Hoerauf, A. (2010). Transforming growth factor-beta expression by host cells is elicited locally by the filarial nematode Onchocerca volvulus in hyporeactive patients independently from Wolbachia. Microbes and Infection $12,555-564$.

Korten, S., Wildenburg, G., Darge, K. and Büttner, D. W. (1998). Mast cells in onchocercomas from patients with hyperreactive onchocerciasis (sowda). Acta Tropica 70, 217-231.

Koyanagi, T., Noguchi, K., Ootani, A., Inagaki, K., Robbins, R. C. and Mochly-Rosen, D. (2007). Pharmacological inhibition of epsilon PKC suppresses chronic inflammation in murine cardiac transplantation model. Fournal of Molecular and Cellular Cardiology 43, 517-522

Li, M. O., Wan, Y. Y., Sanjabi, S., Robertson, A. K. and Flavell, R. A. (2006). Transforming growth factor-beta regulation of immune responses. Annual Review of Immunology 24, 99-146.

Lund, F. E. (2008). Cytokine-producing B lymphocytes - key regulators of immunity. Current Opinion in Immunology 20, 332-338.

Meyer, C. G., Gallin, M., Erttmann, K. D., Brattig, N., Schnittger, L., Gelhaus, A., Tannich, E., Begovich, A. B., Erlich, H. A. and Horstmann, R. D. (1994). HLA-D alleles associated with generalized 
disease, localized disease, and putative immunity in Onchocerca volvulus infection. Proceedings of the National Academy of Sciences, USA 91, 7515-7519.

Muixí, L., Alvarez, I. and Jaraquemada, D. (2008). Peptides presented in vivo by HLA-DR in thyroid autoimmunity. Advanced Immunology 99, 165-209.

Murdoch, M. E. (2010). Onchodermatitis. Current Opinion in Infectious Diseases 23, 124-131.

Murdoch, M. E., Hay, R. J., Mackenzie, C. D., Williams, J. F., Ghalib, H. W., Cousens, S., Abiose, A. and Jones, B. R. (1993). A clinical classification and grading system of the cutaneous changes in onchocerciasis. British Fournal of Dermatology 129, 260-269.

Ottesen, E. A. (1995). Immune responsiveness and the pathogenesis of human onchocerciasis. Fournal of Infectious Diseases 171, 659-671.

Racz, P., Tenner-Racz, K., Luther, B., Buttner, D. W. and Albiez, E. J. (1983). Immunopathologic aspects in human onchocercal lymphadenitis. Bulletin de la Societé de Pathologie Exotique et ses Filiales 76, 676-680.

Satoguina, J. S., Adjobimey, T., Arndts, K., Hoch, J., Oldenburg, J., Layland, L. E. and Hoerauf, A. (2008). Tr1 and naturally occurring regulatory T cells induce IgG4 in B cells through GITR/GITR-L interaction, IL-10 and TGF-beta. European Fournal of Immunology 38, 3101-3113.

Schulz-Key, H. and Soboslay, P. T. (2000). Parasitological and epidemiological aspects of the host-parasite interaction in filariasis and their relevance for intervention and control. Nova Acta Leopoldina 313, 189-204.

Steiner, M. S., Zhou, Z. Z., Tonb, D. C. and Barrack, E. R. (1994). Expression of transforming growth factor-beta 1 in prostate cancer. Endocrinology 135, 2240-2247.

Taylor, A. W. (2009). Review of the activation of TGF-\{beta\} in immunity. Fournal of Leukocyte Biology 85, 29-33.
Taylor, M. D., Harris, A., Nair, M. G., Maizels, R. M. and Allen, J. E. (2006). F4/80 + alternatively activated macrophages control CD4 $+\mathrm{T}$ cell hyporesponsiveness at sites peripheral to filarial infection. Fournal of Immunology 176, 6918-6927.

Timmann, C., Abraha, R. S., Hamelmann, C., Buttner, D. W., Lepping, B., Marfo, Y., Brattig, N. and Horstmann, R. D. (2003). Cutaneous pathology in onchocerciasis associated with pronounced systemic T-helper 2-type responses to Onchocerca volvulus. British Fournal of Dermatology 149, 782-787.

Timmann, C., van der Kamp, E., Kleensang, A., König, I. R., Thye, T., Büttner, D. W., Hamelmann, C., Marfo, Y., Vens, M., Brattig, N., Ziegler, A. and Horstmann, R. D. (2008).

Human genetic resistance to Onchocerca volvulus: evidence for linkage to chromosome $2 \mathrm{p}$ from an autosome-wide scan. Fournal of Infectious Diseases 198, 427-433.

van der Neut Kolfschoten, M., Schuurman, J., Losen, M., Bleeker, W. K., Martínez-Martínez, P., Vermeulen, E., den Bleker, T. H., Wiegman, L., Vink, T., Aarden, L. A., De Baets, M. H., van de Winkel, J. G., Aalberse, R. C. and Parren, P. W. (2007). Anti-inflammatory activity of human IgG4 antibodies by dynamic Fab arm exchange. Science 317, 1554-1557.

Wildenburg, G., Korten, S. and Büttner, D. W. (1998). Mast cell distribution in nodules of Onchocerca volvulus from untreated patients with generalized onchocerciasis. Parasitology 116, 257-268.

World Health Organization (1987). Expert Committee on Onchocerciasis. WHO Technical Report Series No. 752. World Health Organization, Geneva, Switzerland.

World Health Organization (1995). Onchocerciasis and its Control. WHO Technical Report Series No. 852. World Health Organization, Geneva, Switzerland. 